\title{
Strates
}

STRATES Matériaux pour la recherche en sciences sociales

$12 \mid 2006$

Nouvelles tensions impériales et recompositions en Europe centrale, orientale, et CEI

\section{Transformations économiques et nouveaux conflits nationaux dans les Balkans}

\section{Artan FUGA}

\section{OpenEdition}

\section{Journals}

Édition électronique

URL : http://journals.openedition.org/strates/2012

DOI : $10.4000 /$ strates.2012

ISSN : $1777-5442$

Éditeur

Laboratoire Ladyss

Édition imprimée

Date de publication : 31 décembre 2006

ISSN : 0768-8067

Référence électronique

Artan FUGA, «Transformations économiques et nouveaux conflits nationaux dans les Balkans », Strates [En ligne], 12 | 2006, mis en ligne le 20 juillet 2007, consulté le 08 septembre 2020. URL : http:// journals.openedition.org/strates/2012 ; DOI : https://doi.org/10.4000/strates.2012

Ce document a été généré automatiquement le 8 septembre 2020

Tous droits réservés 


\title{
Transformations économiques et nouveaux conflits nationaux dans les Balkans
}

\author{
Artan FUGA
}

1 Après la chute des régimes communistes dans la péninsule balkanique, la vie politique et sociale a été marquée durant la dernière décennie par de nombreux conflits entre les diverses nations et ethnies. Le morcellement de l'ancienne République fédérative yougoslave (RFY) fondée par Tito, les tensions et les combats qui ont accompagné la création de la Slovénie, de la Croatie et de la Macédoine en tant qu'États indépendants, les conflits sanglants entre musulmans, Croates et Serbes en Bosnie-Herzégovine, les interminables rivalités ethniques entre Macédoniens et Albanais en RFY de la Macédoine, la crise du Kosovo de l'an 1999 sont parmi d'autres les événements qui ont marqué l'histoire de cette région.

2 Diverses lectures de ces conflits ont été faites. Cependant, sans négliger l'apport des méthodologies fondées sur des principes politiques, culturalistes, anthropologiques, etc., l'influence des transformations économiques sur l'aggravation des crises et des conflits ethniques ne semble pas toujours étudiée à sa juste mesure. Or, nous pensons que cet aspect doit être particulièrement pris en compte afin de comprendre non seulement la prolongation des anciens conflits ethniques existant dans la région, mais aussi l'émergence de formes nouvelles de conflit liées à la conjoncture socioéconomique actuelle. La mise en exergue des relations entre la sphère économique et la sphère nationale apporte des éléments permettant d'enrichir l'analyse des dynamiques nationales, territoriales et géopolitiques qui caractérisent cette région aujourd'hui.

Du marché national à l'« économie ethnique »

3 Durant les trois derniers siècles, l'enchevêtrement des éléments de la vie économique et des dynamiques nationales peut être découpé en trois grandes périodes.

4 La première caractérise l'époque de la création des marchés nationaux dans l'ensemble des pays balkaniques en dépit des décalages temporels liés à la rapidité de ce processus. Ce fait coïncide avec la création des élites politiques et économiques nationales, la 
constitution graduelle d'une conscience nationale plus ou moins développée et l'existence de stratégies nationalistes basée sur la définition des frontières de chaque pays créé comme État national. À cette liste il faut ajouter des ensembles politicoadministratifs multinationaux constitués au cours $d u x{ }^{e}$ siècle et au début $d u x^{e}$ siècle.

5 La deuxième période historique va de la fin de la Seconde Guerre mondiale au début des années 1990. Sous la coupe de régimes de type communiste et centralisateur, la plupart des pays balkaniques ont géré leurs affaires économiques au moyen d'une étatisation forcée, voire complète, des rapports économiques. En tant que représentant de la nation ou d'une multitude de nations, l'État prétendait contrôler toute la vie économique en appliquant des méthodes autoritaires et dirigistes.

6 La troisième période est la période actuelle. La plupart des nations balkaniques ont recréé leur propre État national. Cette nouvelle réalité politique va de pair avec une libéralisation continue des rapports économiques provenant d'une privatisation générale des moyens de production. L'État abandonne toujours plus la sphère des rapports économiques. Les marchés sont ouverts dans le contexte de la globalisation de la vie économique de notre planète. Les échanges économiques et commerciaux entre les pays de la péninsule ont considérablement augmenté. Cependant, l'un des signes de l'évolution actuelle des sociétés balkaniques est que les politiques menées visent à renforcer l'identité nationale de chaque groupe ethnique local considéré comme prioritaire. Des groupes ethniques et nationaux divers, échappant désormais au contrôle homogénéisant de l'ancien pouvoir totalitaire, vivant parfois sur le même territoire politique et administratif, déploient de nouvelles stratégies politiques, économiques et culturelles en fonction de l'actualité mondiale.

Groupes ethniques et groupes socioprofessionnels

7 Plusieurs auteurs attestent qu'au cours de l'histoire, des rapports très complexes se sont créés entre l'identité nationale d'un peuple et ses activités économiques. On considère généralement que les rapports nationaux subissent l'influence des processus socio-économiques et peuvent générer des dynamiques socio-économiques spécifiques, portant ainsi l'empreinte de la configuration identitaire de chaque nation ${ }^{1}$.

Le livre de Michel Roux, Les Albanais en Yougoslavie (1992), illustre ce propos avec une analyse approfondie et très pertinente des rapports nationaux en Yougoslavie pendant la période qui précède la chute du mur de Berlin. Il rapporte en effet des éléments précieux pour comprendre comment les groupes socioprofessionnels distincts peuvent être constitués majoritairement d'individus appartenant aux divers groupes nationaux $^{2}$. Parfois, la logique de répartition $d u$ travail et des tâches socioprofessionnelles dans le cadre d'un pays multinational se structure selon la configuration des rapports nationaux. Nous qualifions cette réalité d'«économie ethnique ».

9 Il peut arriver qu'un ou plusieurs groupes nationaux soient contraints de ne développer que des types restreints d'activité économique en raison d'un rapport historique déterminé par la logique respectant la dichotomie : nation dominante/ethnie dominée. Une minorité nationale est parfois poussée par la force des choses vers une activité professionnelle considérée comme moins fructueuse économiquement parlant, plus pénible sur le plan physique et moins épanouissante sur le plan intellectuel par les membres de la nation dominante. 
10 Il peut arriver aussi qu'une certaine tradition culturelle, des mœurs et un type précis d'organisation familiale soient plus adaptés à l'exercice d'une quelconque activité économique.

11 Enfin, la reproduction de la configuration économico-ethnique d'une société pérennise son propre relief en orientant, parfois de façon irréversible, le flux des capitaux, l'ensemble des capacités professionnelles des individus, la structure des investissements réalisés dans le monde économique, etc.

12 M. Roux montre, à travers un ensemble de données statistiques riches, qu'en Yougoslavie, avant la chute du mur de Berlin, il y avait une répartition relativement déséquilibrée des individus appartenant à des groupes nationaux distincts dans les principales activités économiques du pays. En consultant les statistiques de l'époque, il constate, par exemple, qu'en Macédoine, $51,3 \%$ des Albanais travaillaient dans l'agriculture, tandis que seulement $29 \%$ des Macédoniens exerçaient la profession d'agriculteur. Un autre exemple: au Monténégro, 31,6\% des Albanais étaient embauchés dans le secteur tertiaire pour $47,4 \%$ de Monténégrins. Dans le cadre fédératif, il y avait seulement $6,9 \%$ des Albanais intégrés professionnellement dans l'administration, tandis que $16 \%$ des Slovènes et $10,5 \%$ des Serbes exerçaient divers métiers dans ce secteur. Le taux d'activité professionnelle des Albanais était de 21,4\%, tandis que celui des Serbes était de $47,6 \%$, des Croates $41,4 \%$, des Slovènes $47,2 \%$, etc. Les traces de cette «économie ethnique » étaient donc bien présentes dans la société yougoslave de l'époque de Tito.

13 Cette structure nationalo-professionnelle s'est trouvée bouleversée après l'effondrement des régimes communistes. Les réformes initiées au cours du processus de structuration économique, de la privatisation, de la restructuration des logiques de production et des services, de la monétarisation de l'économie, etc., ont apporté une nouvelle configuration des rapports nationaux. Elles ont exigé la reformulation des structures territoriales et des rapports politiques de la société. La restructuration des rapports économiques et la recomposition des relations entre divers domaines d'activité professionnelle affectent aussi les relations entre les groupes nationaux en tant que groupes socioprofessionnels relativement distincts. Plusieurs activités économiques engageant le travail des minorités nationales, marginalisées dans le passé, sont devenues des activités économiques relativement importantes sur un fond de crise économique et d'effondrement général des structures de production. Par conséquent, on constate le renforcement de la position économique de la minorité nationaloprofessionnelle marginalisée dans le passé, en l'occurrence, la minorité albanaise.

14 Évidemment, la crise des rapports nationaux n'est pas le simple résultat de l'aggravation de la situation économique des pays balkaniques. Elle accompagne, en effet, la réorganisation de la configuration économique des pays en question et le nouvel équilibre précaire et instable entre divers domaines du secteur économique des sociétés concernées. Aujourd'hui, le groupe minoritaire nationalo-professionnel albanais, marginalisé dans le passé, est devenu plus fort, voire dominant, au plan économique, tandis que le pouvoir politico-administratif reste toujours entre les mains de la nation dominante. Celle-ci continue à garder ses positions grâce au nombre supérieur de ses représentants dans les structures institutionnelles et administratives du pays. De plus, l'avantage numérique de ce groupe albanais le transforme en un groupe électoral dominant, peu en mesure de contrôler les institutions politiques du pays, fondées sur une légitimité historique provenant des assemblées législatives issues 
des élections libres et générales. Par conséquent, ce conflit entre le pouvoir économique et le pouvoir politique prend la forme d'une crise entre la société civile et l'État et d'un déséquilibre entre l'aspect économique et l'aspect administrativobureaucratique de la société. Le projet d'émancipation politique de la minorité nationale, renforcée sur le plan social et économique, se heurte aux résistances du groupe national majoritaire qui veut toujours garder ses positions dominantes au plan politique et militaire. Ainsi, le conflit social s'inscrit sur un terrain de rivalité nationale. Il s'agit d'une configuration déjà ancienne de la structuration des rapports entre divers groupes ethniques constituant la société, mais celle-ci acquiert un nouveau contenu socio-économique et politique dans le contexte actuel.

L'idéologie nationaliste et le mouvement national deviennent des réponses préfabriquées aux questions posées par la restructuration socio-économique du pays. Les minorités nationales traversent une période qui permet leur "épanouissement " économique relatif sur un fond de crise économique généralisée. Avec un statut économique en nette amélioration, face aux dégradations des conditions de vie et d'existence $d u$ groupe national majoritaire, anciennement dominant sur le plan économique et politique, les minorités nationales "relèvent la tête ", résistent aux politiques conduites par les élites dominantes d'une époque révolue et développent des stratégies politiques allant jusqu'à la revendication de la fondation de leur État indépendant.

Afin de décrire cette tendance, nous présentons certains aspects qui la caractérisent.

17 Premièrement, nous distinguons le processus d'effondrement des structures de production industrielle des pays balkaniques et de reformulation des rapports économiques. La crise du secteur industriel est bien plus importante que celle qui caractérise l'agriculture, l'artisanat et les services où la petite propriété résiste mieux aux changements et aux défis de la concurrence. La crise de l'industrie locale frappe d'abord la nation dominante et affaiblit considérablement son élite économique qui constate que le secteur qu'elle contrôlait depuis longtemps n'a plus de rôle prépondérant dans le cadre de l'économie locale. Par contre, les secteurs tertiaires, marginalisés dans le passé, l'artisanat familial, l'agriculture vivrière, le petit commerce, etc., résistent mieux aux changements et donnent un pouvoir économique stable et accru aux individus appartenant à la minorité nationale anciennement marginalisée. De plus, l'effondrement des structures de production industrielle rend le budget de l'État déficitaire et réduit l'action de l'État dans le domaine social ${ }^{3}$.

Deuxièmement, le territoire étatique perd beaucoup de sa cohésion et de son unité économique. Il devient considérablement fragmentaire en développant des structures locales de production, parfois entièrement décentralisées, qui obéissent à des dynamiques totalement nouvelles. La décentralisation du champ des intérêts économiques rend inopérante l'action unificatrice de l'État central, affaiblit le rôle unificateur de la nation dominante, élargit les marges de libertés pour les actions politiques de la minorité nationale localement installée ${ }^{4}$.

19 La cohésion du territoire national ne dépend plus des dynamiques économiques, mais plutôt des relations de pouvoir et du contrôle politique que l'État a de plus en plus de mal à exercer de façon efficace. La zone rurale de ces pays développe des structures de production autogestionnaires et autosuffisantes. Cette zone rurale, lieu privilégié de la minorité nationale marginalisée dans le passé, n’a presque plus besoin de la ville alors que celle-ci était un acteur économique principal pendant l'époque communiste. La 
ville, dorénavant, consomme des produits importés de l'étranger et n'a presque plus besoin de la zone rurale située autour d'elle. Hors du contrôle politique et économique relatif de la ville, les ruraux se constituent en tant qu'acteurs politiques indépendants et tentent de s'imposer aux citadins. Or, ces ruraux, souvent, ne sont autres que les membres de la communauté nationale minoritaire. Ils utilisent l'idéologie nationaliste et le projet politique nationaliste comme idéologie et structure d'action pour essayer de reconfigurer les rapports entre la zone urbaine et la zone rurale du pays imposés par les nouvelles dynamiques économiques de libéralisation. Cela peut expliquer le fait que les mouvements nationalistes radicaux ne se montrent pas seulement hostiles aux structures de l'État qui expriment prioritairement les intérêts de la nation dominante. Ils essayent aussi de faire sortir de la scène politique les élites citadines qui ont représenté traditionnellement la minorité nationale dans les institutions de l'État. Ces dernières se voient accusées d'avoir adopté des politiques nationalistes trop prudentes vis-à-vis de l'État. Cela est facilement vérifiable empiriquement au Kosovo et en Macédoine où les membres des UCK (Ushtrisë të çlirimtare të Kosovës [Mouvement indépendantiste kosovar]) respectifs sont issus majoritairement de la zone rurale et très méfiants à l'égard des structures des partis politiques traditionnels des Albanais.

Troisièmement, il se produit un déséquilibre socioprofessionnel et économique important entre la couche des administrateurs, des responsables économiques, de la bureaucratie d'État, des employés de l'État, des militaires, etc., et les autres professions marginalisées dans le passé, exercées par des individus faisant partie de la minorité nationale. L'État réduit au chômage nombre de ses employés. Il exerce une pression permanente sur les salaires des membres de la fonction publique suite au déficit budgétaire énorme qui marginalise économiquement les anciens membres de l'élite politique dominante. Même l'armée n'a plus sa place prépondérante dans le fonctionnement de la vie sociale parce que son rôle va en se réduisant pendant cette période de détente internationale. L'armée risque aussi de subir une réduction drastique de ses moyens. La crise économique touche d'abord l'ancienne élite nationale dominante et met par contre en position relativement prépondérante les professions «marginales » de l'agriculteur, de l'artisan, du petit commerçant. L'ancienne " plèbe » incarnée par la minorité nationale relève la tête et cherche à devenir politiquement dominante, au moment où elle est en passe de le devenir socialement et économiquement. Elle réclame plus de droits dans le contexte politique de son pays, considérant que son statut politique est moralement injuste. L'idéologie nationaliste, le discours national, l'action séparatiste visent ainsi le rééquilibrage des rapports politiques de la société avec les réalités sociales actuelles.

Quatrièmement, les formes d'organisation économique basées sur la production moderne perdent du terrain. Le petit commerce, l'artisanat traditionnel, l'agriculture autarcique reposent sur une logique d'économie familiale. C'est la production domestique qui devient le support principal de l'économie ethnique. La famille rurale, marquée par une culture clanique, de confession musulmane, préserve mieux la cohésion du groupe familial élargi. Elle s'avère plus adaptée à la production familiale artisanale ou de petit commerce. Par contre, l'ancien collectif salarial se dissout toujours plus et avec lui s'effondrent aussi les anciens liens socioprofessionnels de la nation dominante. Cela se produit durant une période où le groupe socioprofessionnel familial déploie toujours plus intensivement ses fonctions économiques majeures renforçant ainsi les liens entre les membres de la minorité nationale. Dans les marchés des grandes villes de la région, à Tirana, Skopje, Tétovo, Podgorica, etc., on remarque 
parfois que seuls sont mis en vente les produits de consommation provenant de l'étranger ou les produits créés par l'artisanat traditionnel, fabriqués et mis en vente par les acteurs de cette économie ethnique.

Cinquièmement, dans le passé, les membres de la minorité nationale émigraient d'une façon plus marquée. Occupant une position économique précaire dans leur propre pays, ils étaient obligés d'aller travailler et vivre en Occident, créant par conséquent une communauté nombreuse et consolidée à l'étranger. Aujourd'hui où la vie économique du pays se libéralise, les membres de la diaspora arrivent avec de l'argent, ce qui s'avère un élément très important dans le contexte de la crise financière du pays. Ils sont en mesure d'apporter à l'économie familiale le capital dont elle a besoin, de subventionner les partis nationalistes locaux, de se présenter au marché des ventes aux enchères pour acheter des unités de production et de services privatisées dans le contexte des réformes ${ }^{5}$.

Frontières spatiales et frontières ethniques du marché

23 L'économie devient "ethnique " parce que le flux des capitaux et la circulation des marchandises et des services suit des réseaux constitués au sein de chaque communauté ethnique. Les salaires, le recrutement de la main-d'œuvre, la rentabilité financière de l'entreprise, le taux de profit, etc., se calculent à partir d'une logique qui sert à renforcer le groupe national en question. Cette logique ne considère le groupe national adversaire que comme une éventuelle clientèle et absolument pas comme un partenaire. Les membres du groupe national adverse sont peut-être tolérés comme acheteurs, mais non comme vendeurs ${ }^{6}$.

24 Le contenu du concept d'« économie ethnique » est centré sur l'idée que les groupes nationaux et ethniques dans les Balkans, après l'effondrement des politiques autoritaires et centralisatrices en matière d'économie, durant le processus de création des marchés ouverts et supranationaux, construisent leurs propres espaces économiques informels qui représentent des aires de relations de production et d'échange structurés selon une configuration spatiale et relationnelle des rapports ethniques. Chaque nation ou groupe ethnique est orienté vers la création de sa zone économique propre caractérisée par deux éléments principaux : a) un espace territorial où le groupe ethnique ou national est installé ; b) un ensemble de relations sociales à l'intérieur du groupe ethnique ou national qui n'est pas forcément inscrit dans un espace continu et homogène. Les membres d'un même groupe national ou ethnique peuvent entretenir des relations économiques et commerciales privilégiées entre eux sans être installés sur le même espace administratif ou sur la même aire géographique. Cependant, les distances géographiques ne les empêchent pas de former une communauté économique et une zone commerciale cohérente, unique et souvent fermée sur elle-même. Les groupes ethniques et nationaux ont tendance à faire circuler les biens, les marchandises, les produits, les services, les capitaux, la main-d'œuvre, etc., dans des circuits déterminés par leur identité nationale.

Dans ce contexte, la rationalité proprement économique se structure dans le cadre de la réalisation des stratégies politiques d'affirmation nationale. Les anciennes frontières économiques étanches entre les États autoritaires lors de l'époque communiste risquent de perdurer en adoptant de nouvelles formes. Elles ne marquent plus des coupures spatiales sur des territoires géographiques. Se développant parfois sur le territoire d'une même ville et d'une même région, ces frontières sont déjà portées par un support humain, dispersé sur un espace territorial assez flou et entremêlé avec des 
populations ayant une tout autre identité nationale. Le système de partenariat économique suit le plus souvent plutôt une logique nationale que purement économique. Dans ce contexte domine une mentalité de ségrégation : "Mon voisin ", dit-on, n'est plus celui qui vit et travaille à côté de moi, mais mon « confrère » national là où il vit et où il exerce son métier. Au niveau spatial, le marché économique correspond à l'étendue de la dispersion des individus appartenant à la même nation ou à la même ethnie. Au sein d'une structure économique étatique globalisée, existent en effet au moins deux ou plusieurs «économies ethniques » ayant leurs propres logiques de dispersion et de fonctionnement. Loin de permettre la cohésion sociale du pays, cette " économie ethnique ", même si elle se développe progressivement, renforce les clivages ethniques et nationaux qui déchirent le corps social tout entier et préparent les conflits du futur.

\section{BIBLIOGRAPHIE}

Chiclet Ch., Lory B. (dir.), 1998, La république de Macédoine. Nouvelle venue dans le concert européen, Paris, L'Harmattan.

Chiclet Ch., Slim A., 2000, « Macédoine 1999-2000 », Le Courrier des pays de l’Est, n 1006, juinjuillet.

Del Valle A., 2000, Guerres contre l'Europe, Paris, Éditions des Syrtes.

Fuga A., 2000, Identités périphériques en Albanie, Paris, L'Harmattan.

Fuga A., Lhomel E., 2000, « Albanie. 1999-2000, Zizanie politiques, paradoxes économiques », Le Courrier des pays de l'Est, $\mathrm{n}^{\circ} 1006$, juin-juillet.

Fuga A., 2003, Les mots dans la communication politique en Albanie, Paris, l'Harmattan.

Heimeri D., 2000, « Serbie-Monténégro 1999-2000. Échec et mat? », Le Courrier des pays de l'Est, $\mathrm{n}^{\circ} 1006$, juin-juillet.

Rauffer X., Haut F., 1992, Le Chaos balkanique, Paris, La Table Ronde.

Rey V. (dir.), 1993, Géographie et Campagnes, Paris, École normale supérieure de Fontenay/Saint Cloud.

Roux M., 1992, Les Albanais en Yougoslavie, Paris, Fondation de la Maison des sciences de l'homme.

Rugova I., 1994, La Question du Kosovo, Paris, Fayard.

\section{NOTES}

1. 1. Nous avons trouvé cette analyse dans le livre de M. Roux (1992). Il y affirme clairement : «J'ai tenté de dépasser l'économisme qui caractérise, en particulier dans les pays de l'Est, le traitement des problèmes de développement régional et d'envisager dans leurs relations dialectiques les deux ordres de problèmes déjà évoqués : ceux qui 
concernent l'économie (politiques de développement, transferts de ressources, croissance et différenciation socio-économique de l'espace) et ceux qui touchent aux relations des nationalités entre elles et avec l'État (localisation du pouvoir, marquage symbolique, organisation et contrôle effectif du territoire, rapports de forces politiques et mode de règlement des conflits). Le rapprochement de ces deux thématiques permet d'aborder une problématique complexe de l'intégration régionale » (p. 17).

2. V. Rey a formulé cette approche en l'appliquant à l'espace de l'Europe médiane. Dans son article « Nation et État, deux structures rivales en région de sémi-périphérie - le cas de l'Europe centre-orientale ", elle écrit : « Les colonies germaniques de mineurs et d'artisans ont été le terreau des bourgeoisies industrielles locales ; les marchands juifs, grecs et arméniens ont assuré le commerce, la collecte des impôts et des rentes foncières, les soldats islamisés ont gardé les marges de l'empire ottoman, les esclaves tsiganes ont constitué la main-d'œuvre de grands domaines, etc. Tout cela a bloqué la différenciation sociale à l'intérieur de chaque nation, tandis que l'émergence des conflits de classes s'exprimait en conflits de peuples antagonistes sur un même espace. La progressive constitution des peuples en nations les a poussés à revendiquer comme territoire propre ce qui auparavant était lieu commun » (Rey, 1993, p. 28).

3. Je renvoie le lecteur à deux articles afin qu'il puisse comprendre mieux les dynamiques de l'effondrement économique dans les pays balkaniques, particulièrement en Macédoine, au Monténégro et en Serbie : C. Chiclet et A. Slim, 2000.

4. Il est, peut-être, important de rapporter ici les propos d'I. Rugova affirmant cette fragmentation économique du territoire du pays qui facilite l'action des nationalistes. Il dit: " Je dirais même que nous tenons de mieux en mieux. Nous sommes en train de prouver que nous sommes capables de vivre indépendamment d'autres régions de l'exYougoslavie. Que nous avons aussi une sorte d'indépendance économique de fait » (I. Rugova, 1994).

5. I. Rugova (1994) affirme : « La diaspora arrive à nous faire parvenir de l'aide. Ce n'est pas une diaspora politique, comme celle qui avait émigré vers les États-Unis, la France ou l'Angleterre après la Seconde Guerre mondiale et qui était formée de groupes élitistes qui n'ont pas beaucoup réussi dans le commerce. Aujourd'hui, la diaspora est plutôt constituée de travailleurs qui aident leurs familles et nous soutiennent financièrement - politiquement aussi ».

6. À Skopje, il y a deux têtes de station de taxis près du marché de la ville, une où stationnent les taxis albanais servant les Albanais, et l'autre pour les taxis macédoniens et au service des Macédoniens. À Tetovo, les Albanais fréquentent les restaurants tenus par leurs compatriotes, tandis que les Macédoniens vont dans les cafés et les restaurants ayant comme patrons des personnes d'origine macédonienne. Les Albanais du Kosovo et de la Macédoine passent souvent leurs vacances sur les côtes maritimes de l'Albanie où ils ouvrent pendant l'été des restaurants, des commerces et des locaux de service. Au Kosovo, il est très difficile pour un patron d'une petite entreprise d'embaucher des individus appartenant à un autre groupe national que le sien. 


\section{RÉSUMÉS}

Les conflits ethniques dans les Balkans se sont multipliés durant les deux précédentes décades. Des approches variées ont été développées et ont essayé de leur trouver une explication. Sans négliger l'importance des approches culturalistes et géoplitiques, cet article présente un point de vue basé sur le concept «d'économie ethnique ». L'hypothèse proposée est que la croissance des conflits nationaux dans l'espace occupé par l'ex-Yougoslavie est fortement reliée aux changements profonds intervenus dans les économies nationales, sur une base ethnique repérable, dans la perspective de la globalisation, de la privatisation et de la libéralisation des relations économiques nationales. Dans cette perspective, l'hypothèse formulée est que dans les conditions présentes, il y a une profonde contradiction entre le fait que l'économie et les biens matériels tombent de façon croissante dans les mains de nations auparavant opprimées, tandis que les structures étatiques et de pouvoir restent aux mains des nations traditionnellement dominantes. Cette hypothèse autorise une nouvelle interprétation des situations qui se font jour au Kosovo et en Macédoine.

Artan Fuga. Economic transformations and new national conflicts in the Balkans

Ethnic conflicts in the Balkan region have multiplied during the past two decades. Various approaches have been developed to try and explain them. Without neglecting the importance of the culturalist or the geopolitical approach, this article presents a point of view based on the concept of ethnic economy. The hypothesis is that the growth of national conflicts in the space occupied by former Yugoslavia is strongly linked to deep changes in national economies grounded on an ethnic basis, in a perspective of globalisation, privatisation and liberalisation of national economic relationships. In this perspective, the hypothesis put forward is that in the present conditions, there is a deep contradiction between the fact that the economy and material goods are increasingly falling in the hands of nations formerly oppressed, whilst the state structures and political power remain with the traditionally dominant nations. This hypothesis allows a new and euristic interpretation of the case of Kosovo and Macedonia.

\section{AUTEUR}

\section{ARTAN FUGA}

Professeur à l'université de Tirana, chercheur associé au Ladyss, artan.fuga@libertysurf.fr 